\title{
Prognosis following acute coronary syndromes according to prior coronary artery bypass grafting: Meta-analysis
}

$1-10$

(c) The European Society of Cardiology 2014

Reprints and permissions: sagepub.co.uk/journalsPermissions.nav DOI: |0.| |77/20488726|4554| I0 acc.sagepub.com

(S)SAGE

\author{
Rogério Teixeira',2, Maria J Vieira², Miguel A Ribeiro', Lino \\ Gonçalves ${ }^{2,3}$ and Bernard J Gersh ${ }^{4}$
}

\begin{abstract}
Purpose: Conduct a meta-analysis to study the prognostic influence of a previous coronary artery bypass grafting (CABG) in patients admitted for an acute coronary syndrome (ACS).

Methods: A systematic review of the literature was performed using electronic reference databases through January 2013 (MEDLINE, Cochrane Library, Web of Knowledge, Google Scholar and references cited in other studies). Studies in which ACS outcomes with a previous history of CABG were compared with ACS outcomes with no history of previous CABG were considered for inclusion. The main endpoints of interest were mortality and non-fatal acute myocardial infarction. Data was aggregated at three follow-up times using random-effects meta-analysis models.

Results: Twenty-four studies were included which provided 387,181 patients for analysis. Previous CABG ACS patients were older, more diabetic and had a more frequent history of a previous myocardial infarction. Pooled in-hospital mortality was higher for the previous CABG ACS patients (OR I.22 [I.04-I.44], $p<0.01,1288 \%$ ). The pooled adjusted OR showed no significant differences for the two groups (adjusted OR I.I3 [0.93-1.37], $p=0.22$, I2 92\%). Previous CABG ACS patient had a higher pooled 30-day mortality (OR I.28 [I.05-I.55], $p=0.02$, I2 74\%); a higher non-adjusted (OR I.6I [I.38-1.88], $p<0.01, \mathrm{I}^{2} 70 \%$ ) and adjusted (adjusted OR I.37 [I.I5-1.65], $p<0.01, \mathrm{I}^{2} 0 \%$ ) long-term mortality. Both the in-hospital and the long-term re-infarction rates were higher for the previous CABG ACS patients.

Conclusions: According to our data, ACS patients with previous CABG history had a higher risk for short- and longterm adverse events.
\end{abstract}

\section{Keywords}

Acute coronary syndromes, coronary artery bypass grafting, meta-analysis, prognosis

Date received: 18 August 2014; accepted: 14 September 2014

\section{Introduction}

Coronary artery bypass grafting $(\mathrm{CABG})$ has been an established therapy for patients with obstructive coronary artery disease since the 1970 s, with a major impact on symptoms ${ }^{1}$ and in some subsets on mortality and subsequent cardiovascular events. ${ }^{2}$ Until the late 1990s there had been increasing numbers of CABGs performed annually, in Europe and in the USA. ${ }^{3}$ This, along with the progression of native coronary atherosclerosis and saphenous vein graft disease during long-term follow-up, has led to an increase in the proportion of patients with an acute coronary syndrome (ACS) who have previously undergone CABG. ${ }^{4}$

Post-CABG patients presenting with acute myocardial infarction (MI) have been shown to be older, with a greater proportion of diabetes, heart failure, and previous MI. ${ }^{5,6}$ Nonetheless it has been demonstrated that they usually

'Departamento de Medicina, Serviço de Cardiologia, Hospital Beatriz Ângelo, Loures, Portugal

${ }^{2}$ Faculdade de Medicina da Universidade de Coimbra, Coimbra, Portugal ${ }^{3}$ Serviço de Cardiologia, Centro Hospitalar e Universitário de Coimbra Hospital Geral, Coimbra, Portugal

${ }^{4}$ Division of Cardiovascular Disease and Internal Medicine, Mayo Clinic, Rochester USA

\section{Corresponding author:}

Rogério Paiva Cardoso Teixeira, Departamento de Medicina, Serviço de Cardiologia, Avenida Carlos Teixeira - 3, 2674-5I4 Loures, Portugal. Email: rogeriopteixeira@gmail.com 
have smaller infarcts and a lower incidence of Q-wave MIs. 7,8 Therefore it has been hypothesized that a prior CABG could exert a protective effect, as a result of the double coronary circulation and ischemic preconditioning. ${ }^{6,9,10}$

The purpose of this meta-analysis was to systematically review and synthesize existing data on prognosis of patients with ACS and a history of a previous $\mathrm{CABG}$, with respect to in-hospital, short-term and long-term outcomes.

\section{Methods}

\section{Search strategy for the identification of studies}

We searched electronic databases for articles published in English until January 2013. Databases included MEDLINE, Cochrane Library (Cochrane Central Register of Controlled Trials), Web of Knowledge, Google Scholar and references cited in other studies. The medical subject headings and keyword searches included the terms "acute coronary syndrome", "acute myocardial infarction", "prior coronary arterial bypass". Citations were screened at the title/abstract level and full texts were manually obtained for all potentially relevant articles.

Two evaluators independently extracted all data. Discrepancies were resolved by consensus. Additionally, we signed up with PubMed to receive automated electronic notification for any new articles containing the above search terms.

\section{Criteria for considering studies for this review}

All published studies in which ACS outcomes with a previous history of CABG were compared with ACS outcomes with no history of previous $\mathrm{CABG}$, were considered for inclusion in this meta-analysis. Our pre-specified inclusion criteria were as follows: the studies should compare ACS outcomes among patients with and without prior CABG. Moreover, the ACS should not be related to the immediate postoperative period. The study design could have been observational (either single or multicenter and either prospective or retrospective data) or as a post hoc analyzes of randomized controlled trials (RCTs). Exclusion criteria were duplicate publication; ongoing/unpublished study and studies published only as an abstract or in conference proceedings.

\section{Identification}

The MeSH headings strategy yielded 1969 publications. One additional publication was identified after searching the reference lists of the relevant publications. Over 1473 publications were excluded because it was clear from the title and abstract that they did not fulfil the selection criteria. Two reviewers independently selected the studies to be included, in accordance with the described selection criteria, and read the remaining 27 articles in full. Three studies were excluded.7,11,12 Twenty-four studies were finally included in the meta-analysis, see Table 1 and Supplemental Figure 1.

\section{Methods of review}

The following information was extracted from relevant studies: study design, study characteristics, patient characteristics (age, gender, history of diabetes, and previous MI), admission diagnosis, and patient management (invasive/ conservative strategy; in-hospital medical therapy; percutaneous coronary intervention (PCI), fibrinolysis). The endpoints of interest were in-hospital, short-term and long-term total mortality. Short-term included mortality from discharge to 30 days and long-term included mortality from 90 days to 9.5 years of follow-up.

With respect to in-hospital management, we also analyzed the pooled data for cardiogenic shock, stroke, and major bleeding rate for both groups. Re-infarction was also analyzed in two separate results: in-hospital and long-term follow-up that included data from 6 months to 9.5 years of follow-up.

\section{Statistical analysis}

We used RevMan 5.022 (Nordic Cochrane Centre, København, Denmark) to estimate pooled odds ratio (OR) based on random effects model meta-analysis (inverse variance method). We assumed similarity between the relative risk and OR. Whenever possible we used pooled adjusted relative risk or OR from the primary studies; otherwise, we used raw outcome data to yield unadjusted the ORs. The standard error of the adjusted ORs was calculated according to the formula: [n(upper $95 \% \mathrm{CI})-\ln (\mathrm{OR})] / 1.96$. In view of the potential diversity of study designs, we chose to stratify data for inhospital mortality, based on admission diagnosis and the type of study. The following grouping was elaborated: i) admission diagnosis: studies that included only ST- or only non-ST-elevation ACS patients; ii) study design: stratification was done for post hoc RCTs and post hoc multicenter registries data; and iii) studies publication date: publication before 2000; publication between 2000 and 2010; publication after 2010. Statistical heterogeneity was assessed using the $\mathrm{I}^{2}$ statistic, and values of 30 $60 \%$ represented a moderate level of heterogeneity. Publication bias was estimated visually with funnel plots.

\section{Results}

The search results yielded 24 relevant studies with 387,181 patients. They comprised nine post hoc analyses of RCTs, 11 multicenter registries, and four single centre studies. The main characteristics of the included studies are described in Table 1. 


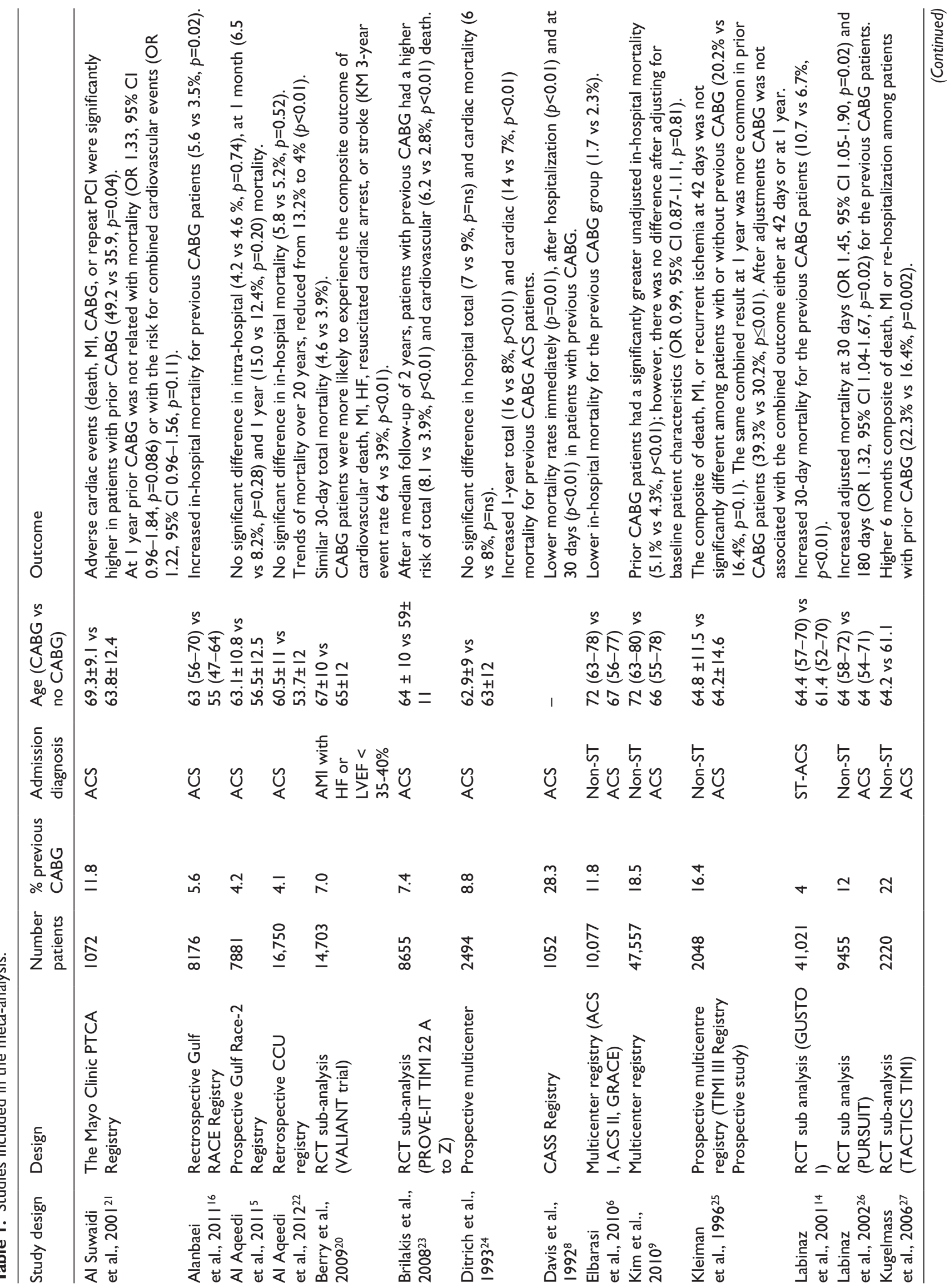




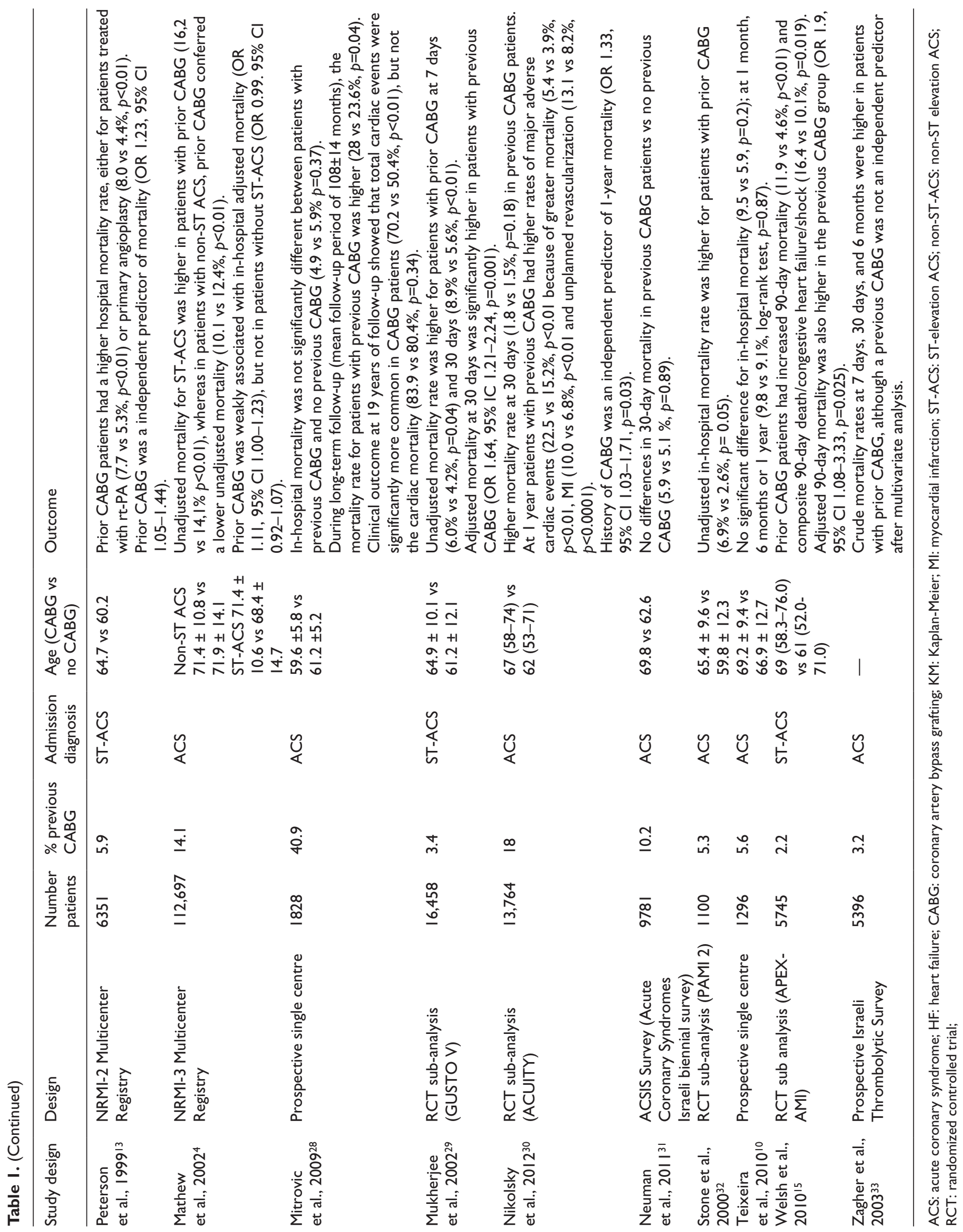


Table 2. Pooled baseline characteristics and in-hospital medical therapy.

\begin{tabular}{|c|c|c|c|c|}
\hline & \multirow[t]{2}{*}{ Mean difference } & \multicolumn{2}{|c|}{ Number of patients included } & \multirow[t]{2}{*}{$1^{2}$} \\
\hline & & $\begin{array}{l}\text { Previous CABG } \\
\text { ACS patients }\end{array}$ & $\begin{array}{l}\text { No previous CABG } \\
\text { ACS patients }\end{array}$ & \\
\hline Age (years) & $2.88(1.35-4.4 I)$ & 20,746 & 166,236 & 99 \\
\hline \multirow[t]{2}{*}{ LVEF (\%) } & $-6.46(-8.7 \mathrm{I}$ to $-4.2 \mathrm{I})$ & 17,206 & 101,539 & 98 \\
\hline & Pooled OR & $\begin{array}{l}\text { Previous CABG } \\
\text { ACS patients }\end{array}$ & $\begin{array}{l}\text { No previous CABG } \\
\text { ACS patients }\end{array}$ & $\mathrm{I}^{2}$ \\
\hline ST-elevation ACS & $0.22(0.11-0.43)$ & $4880 / 18,444$ & $52,547 / 129,998$ & 99 \\
\hline Non-ST-elevation ACS & $3.45(1.96-6.09)$ & $13,458 / 18,444$ & 77,312 / I29,998 & 98 \\
\hline Male gender & I.56 (I.43-I.7I) & $29,818 / 40,392$ & $222,812 / 336,592$ & 90 \\
\hline Diabetes & $1.75(1.57-1.95)$ & I5,373 / 39,344 & 84,1 I 8 / 334,743 & 94 \\
\hline Previous MI & $5.70(4.69-6.94)$ & $21,715 / 39,279$ & $65,685 / 332,165$ & 98 \\
\hline Aspirin & $0.96(0.74-1.23)$ & $|5,84| / 19,364$ & $135,515 / 157,612$ & 95 \\
\hline Second AAP & $0.88(0.71-1.09)$ & $14,070 / 17,627$ & $101,510 / 134,918$ & 94 \\
\hline Ilb/Illa inhibitors & $0.94(0.61-1.44)$ & $1635 / 16,137$ & 16,498 / 97,984 & 97 \\
\hline $\begin{array}{l}\text { Beta-blockers } \\
\text { ACEi / ARA }\end{array}$ & $\begin{array}{l}0.95(0.81-1.10) \\
1.05(0.82-1.36)\end{array}$ & $\begin{array}{l}9767 / 19,236 \\
1116 / 2198\end{array}$ & $\begin{array}{l}80,129 / 151,995 \\
23,556 / 45,094\end{array}$ & $\begin{array}{l}93 \\
83\end{array}$ \\
\hline
\end{tabular}

AAP: antiplatelet therapy; ACEi: angiotensin converting enzyme inhibitors; ACS: acute coronary syndrome; ARA: angiotensin II receptor antagonist; CABG: coronary artery bypass grafting; MI: myocardial infarction; LVEF: left ventricular ejection fraction; I2: study heterogeneity.

Regarding baseline characteristics, the pooled data associated previous CABG ACS patients with an older age (mean difference +2.88 years [1.35-4.41], I2 $99 \%$ ) and with male gender (OR 1.56 [1.43-1.71], I² 90\%). Regarding admission diagnosis, non-ST-elevation ACS was more frequent for patients with a previous CABG history. The pooled data identified a higher prevalence of diabetes (OR 1.75 [1.57-1.95], $\mathrm{I}^{2}$ 94\%) and of previous MI (OR 5.70 [4.69-6.94], $\mathrm{I}^{2} 98 \%$ ) in the CABG ACS patients. Moreover, the previous CABG ACS patients also had a lower pooled left ventricular ejection fraction (mean difference $-6.46 \%$ [-8.71-4.21], I ${ }^{2}$ 98\%) (Table 2).

\section{In-hospital mortality}

Fourteen studies were included in the pooled analysis of inhospital all-cause mortality. During hospital stay 2854 $(8.8 \%)$ of 32,060 previous CABG patients died, compared with $21,354(7.6 \%)$ of 282,603 in the no previous CABG history patients (OR 1.22 [1.04-1.44], $p<0.01, \mathrm{I}^{2} 88 \%$ ) (Figure 1(a)). The difference remained significant for the studies that included only ST-elevation ACS patients (OR 1.39 [1.17-1.65], $p<0.01, \mathrm{I}^{2} 68 \%$ ). For studies that included only non-ST-elevation ACS patients, there were no significant differences between the groups (OR 1.01 [0.70-1.44], $p=0.97, \mathrm{I}^{2} 94 \%$ ) (Supplemental Figure 2(a)). With respect to the design of the study, the pooled data from RCTs identified a worse prognosis for the previous CABG ACS patients (OR 1.36 [1.06-1.73], $p=0.01, \mathrm{I}^{2} 16 \%$ ), that was in contrast with data from observational multicenter registries (OR 1.22 [0.96-1.54], $p=0.1, \mathrm{I}^{2}$ 92\%) (Supplemental Figure 2(b)). Regarding publication date, we noted a worse prognosis for the previous $\mathrm{CABG} A C S$ patients in studies published before 2000 (OR 1.44 [1.04-2.00], $p=0.03, \mathrm{I}^{2} 58 \%$ ). A similar inhospital mortality was noted for studies published between 2000 and 2010, and after 2010 (Supplemental Figure 2(c)). Finally the pooled adjusted OR available for only six trials, with a total of 173,826 patients, concluded that there were no significant differences for the two groups regarding in-hospital adjusted mortality (adjusted OR 1.13 [0.93-1.37], $p=0.22$, $I^{2}$ 92\%) (Figure 1(b)).

\section{In-hospital management and morbidity}

With respect to in-hospital management, ACS patients with a history of a previous CABG had a lower pooled probability of a percutaneous coronary intervention during hospital stay (OR 0.75 [0.63-0.90], $p<0.01, \mathrm{I}^{2}$ 96\%) (Figure 2(a)); and a lower pooled rate of in-hospital fibrinolysis (OR 0.44 [0.34-0.57], $p<0.01, \mathrm{I}^{2}$ 93\%) (Supplemental Figure 3(a)). Cardiogenic shock was significantly more frequent in the previous CABG history patients (OR 1.52 [1.34-1.74], $p<0.01, \mathrm{I}^{2} 0 \%$ ) (Figure 2(b)). Previous CABG patients had a higher rate of in-hospital re-infarction (OR 1.41 [1.091.83 ], $p<0.01, \mathrm{I}^{2} 31 \%$ ) (Figure 2(c)). There were no significant differences between groups with respect to stroke and in-hospital major bleeding rates (Supplemental Figure 3(b) and (c), respectively).

\section{Short and long-term data}

With respect to 30-day mortality, data was available from 12 studies including 130,099 patients. Over 570 of 9374 (6.1\%) previous CABG patients died, in comparison with 6436 


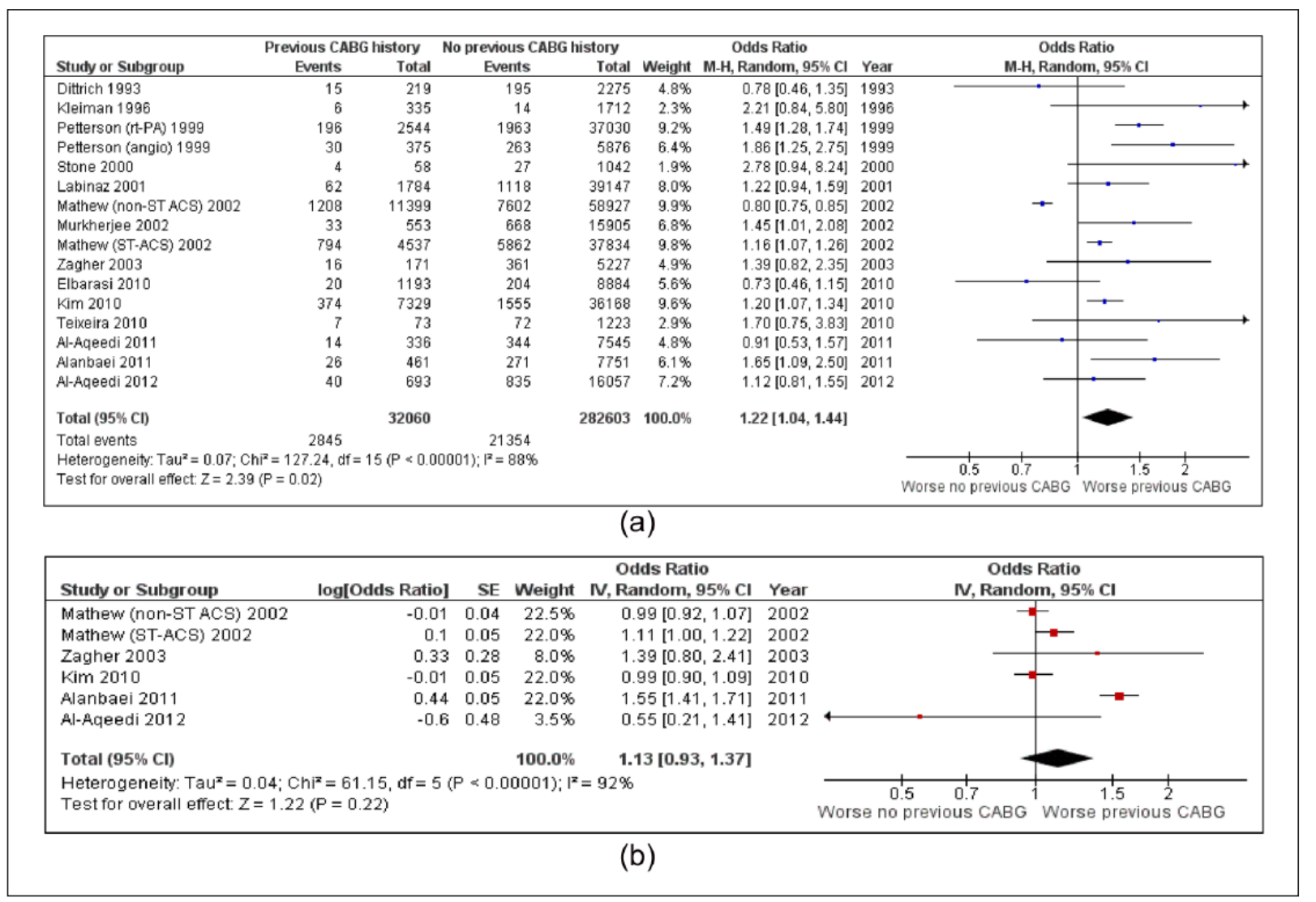

Figure I. In-hospital outcome. (a) Forrest plot for in-hospital mortality. (b) Forrest plot for adjusted in-hospital mortality.

from 11,4619 (5.6\%) with no previous CABG ACS patients. These pooled data was statistically significant (OR 1.28 [1.05-1.55], $p=0.02, \mathrm{I}^{2}$ 74\%) (Supplemental Figure 4(a)). Long-term mortality data was available from 12 studies, with a total of 8590 events. Previous CABG history was associated with a significant worse long-term mortality (OR 1.61 [1.38-1.88], $p<0.01, \mathrm{I}^{2} 70 \%$ ) (Figure 3(a)).

Adjusted data was available for four studies, which allowed an estimation of an adjusted pooled OR. Even after adjustment, previous CABG was associated with a higher rate of long-term mortality (adjusted OR 1.37 [1.15-1.65], $p<0.01, \mathrm{I}^{2} 0 \%$ ) (Figure 3(b)).

With respect to long-term re-infarction rate, data was available for 33,160 patients with a total of 2536 events. Previous CABG history patients were more likely to have a re-infarction during follow-up (OR 1.88 [1.44-2.46], $p<0.01, \mathrm{I}^{2} 80 \%$ ) (Supplemental Figure 4(b)).

\section{Discussion}

According to our pooled data, previous CABG patients admitted for an ACS had a worse prognosis, especially for the long-term.

The impact of prior CABG on survival after an ACS is a controversial subject. Previous studies from the 1990s ${ }^{13}$ and the $2000 \mathrm{~s}^{6,9}$ have suggested that patients with prior bypass surgery have an increased in-hospital mortality, which was likely because of more adverse baseline characteristics in the post-CABG cohort. Our data seem to be in agreement. First, our baseline pooled data associated previous CABG ACS patients with an older age, and with higher risk variables such as diabetes, previous MI, and a lower LVEF. Second, our pooled adjusted OR did not confer an increased in-hospital mortality. This probably means that the higher non-adjusted in-hospital mortality of previous CABG patients was likely the result of older age and multimorbidity, and not the previous surgery itself.

We improved the inconsistency of the data regarding inhospital mortality by stratifying the population. In this way, we were able to conclude that ST-elevation previous CABG ACS patients had higher in-hospital mortality. It has been suggested that post-CABG MIs tend to be smaller in relationship with a number of factors: small vessel disease; collateral circulation or native vessel disease distal to a graft. ${ }^{4}$ Nevertheless, the presence of an ST-segment elevation is suggestive of a larger lesion in a form of transmural myocardial injury and usually represents an acute occlusion of an epicardial coronary artery or of a graft. Contrary to previous reports, ${ }^{14}$ recent data regarding prior CABG ST-elevation ACS patients, reported a nearly equal distribution of the 


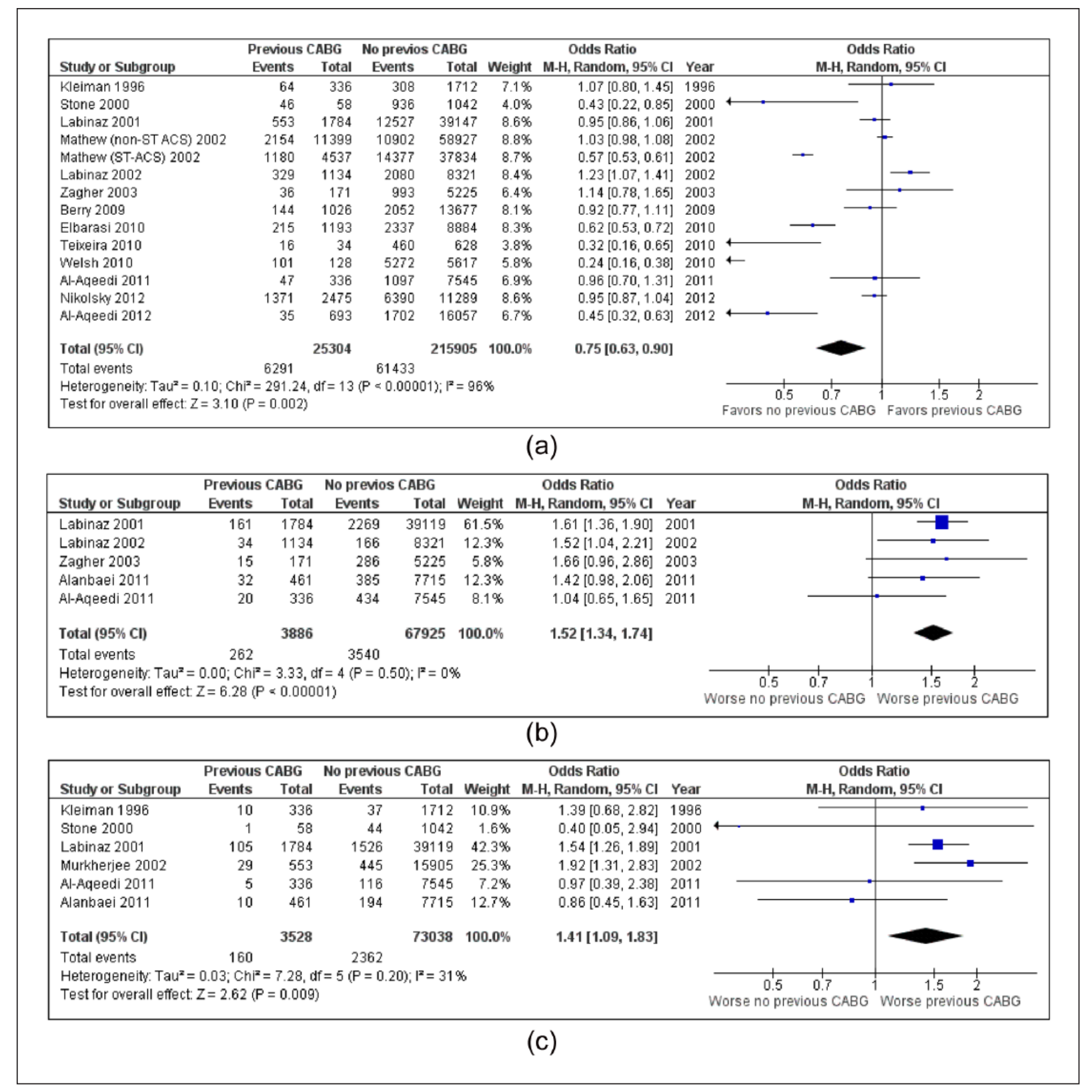

Figure 2. In-hospital management. (a) Forrest plot for in-hospital PCI. (b) Forrest plot for cardiogenic shock. (c) Forrest plot for in-hospital re-infarction.

infarct related artery, between bypass grafts and native coronary vessels. ${ }^{15}$ Moreover, the graft culprit patients had a lower success of primary PCI, and a significantly higher rate of clinical events. In that same trial, over $20 \%$ of prior CABG patients planned for primary PCI, failed to receive reperfusion, mainly because of technical limitations and for the inability to identify a culprit vessel. Those $20 \%$ of patients also had a significantly higher short-term mortality supporting the concept that there is no evidence of a protective effect of a dual coronary circulation in patients who failed to receive primary PCI. ${ }^{15}$ Underutilization of reperfusion therapies in ST-elevation post-CABG patients has also been reported since the lytic era ${ }^{16}$ with significant prognostic implications. ${ }^{14}$ It has also been demonstrated that previous CABG patients have an increased total atherosclerotic burden and more advanced disease overall, as well as a greater clot burden, and longer lesions, which are factors associated with a higher resistance to thrombolytic therapy. ${ }^{14}$ We believe our data indirectly corroborate this worse prognosis of ST-elevation ACS patients, not only because of the higher in-hospital mortality, but also the lower rate of percutaneous intervention and fibrinolysis in the previous CABG ACS patients.

Of interest was that in the non-ST-elevation ACS group of patients, in-hospital mortality was similar between the previous CABG and the rest of the ACS cohort. That was 


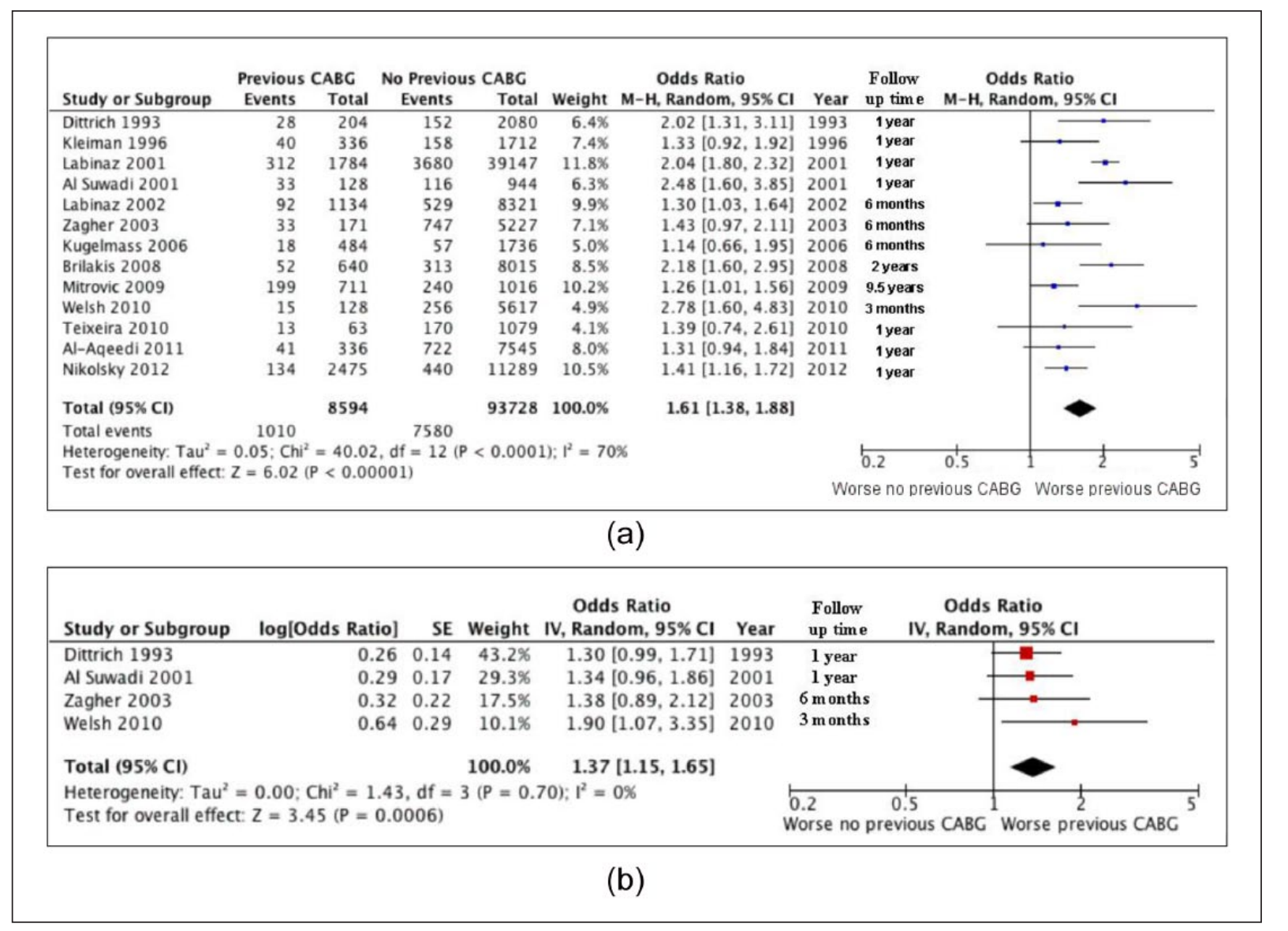

Figure 3. Long-term outcomes. (a) Forrest plot for long-term mortality. (b) Forrest plot for long-term adjusted mortality.

true despite the older age, the higher risk profile, and the more conservative approach. It is certainly plausible that the effect of the dual coronary circulation, the presence of collaterals, and the ischemic preconditioning could be protective. This seems especially important in the context of small non-transmural myocardial ischemic lesions.

Regarding in-hospital major bleeding we concluded, although with a high level of heterogeneity, that major bleeding was not significantly increased for previous CABG patients. Although in the past 20 years optimized medical therapy has improved significantly in the postCABG subset of patients, ${ }^{17}$ recent reports from the present decade concluded for a less aggressive antiplatelet and anticoagulation therapy in those patients, probably protecting them from an increased bleeding. ${ }^{9}$ Moreover, a lower rate of percutaneous intervention and lytic therapy could also have influenced this result.

Our pooled data also demonstrated that percutaneous revascularization was less common in post-CABG patients, in both ST- and non-ST-elevation cohorts. The reasons for this are probably diverse. Previous authors have reported that CABG patients during an ACS are less referred for an invasive strategy ${ }^{4}$ despite their increased ischemic risk, supporting a concept of a paradoxical strategy allocation..$^{18}$ The lower referral could be because of older age, multimorbidity, and previously known coronary anatomy, considered not to be amenable to further revascularization. Also, post-CABG patients referred for an invasive strategy often may not receive such therapy because of complex anatomy and the problems associated with interventions on diseased saphenous vein grafts. ${ }^{15}$ This conservative management, associated with a lower rate of arterial and saphenous graft patency at 10 years after surgery, ${ }^{19}$ and a lower use of medical therapy, ${ }^{20}$ could also be important factors to explain the higher short- and long-term re-infarction rates, and consequently a higher long-term mortality of postCABG patients after an ACS. Unfortunately the majority of the studies did not report time from CABG to the coronary event. This would be important to further stratify data, as the different age of the grafts at the time of infarction could likely exert either a beneficial or deleterious effect.

Post-CABG ACS patients are a complex group of patients, with respect to multimorbidity, coronary anatomy, and revascularization. Our data was consistent with a worse prognosis for this ACS subset of patients, but the outcomes also seem to be influenced by the more adverse baseline 
variables, for which adjustments are not complete. Whether the events in this ACS subset of patients can be prevented by a repeat revascularization procedure is still uncertain. Strategies should continue to be developed to minimize the adverse outcome of this ACS subset of patients, with emphasis on medical therapy, innovative percutaneous treatments, and minimally invasive surgical procedures.

\section{Limitations}

Our analysis is based on studies from the 1990s to 2012 . Methodologies across the studies were not uniform regarding ACS definition, medical therapy, revascularization, and even the surgical technique. We have included in our analysis RCTs sub-analysis and observational (single and multicenter) studies. All of these factors contributed to the heterogeneity in various endpoints, which influenced the consistency of our results. We also suspect the existence of publication bias, as the Funnel plots (Supplemental Figure 5) are not symmetric.

\section{Conclusions}

Post-CABG patients had a worse outcome after an ACS, especially regarding long-term mortality, short- and longterm re-infarction.

\section{Conflict of interest}

None declared.

\section{Funding}

This research received no specific grant from any funding agency in the public, commercial, or not-for-profit sectors.

\section{References}

1. Myers WO, Blackstone EH, Davis K, et al. CASS registry Long term surgical survival. J Am Coll Cardiol 1999; 33(2): 488-98.

2. Shahian DM, O'Brien SM, Sheng S, et al. Predictors of Long-Term Survival After Coronary Artery Bypass Grafting Surgery: Results From the Society of Thoracic Surgeons Adult Cardiac Surgery Database (The ASCERT Study). Circulation 2012; 125(12): 1491-500.

3. Ferguson TB, Jr., Hammill BG, Peterson ED, et al. A decade of change-risk profiles and outcomes for isolated coronary artery bypass grafting procedures, 1990-1999: a report from the STS National Database Committee and the Duke Clinical Research Institute. Society of Thoracic Surgeons. Ann Thorac Surg 2002; 73(2): 480-9.

4. Mathew V, Gersh B, Barron H, et al. Inhospital outcome of acute myocardial infarction in patients with prior coronary artery bypass surgery. Am Heart J 2002; 144(3): 463-9.

5. Al-Aqeedi R, Sulaiman K, Al Suwaidi J, et al. Characteristics, management and outcomes of patients with acute coronary syndrome and prior coronary artery bypass surgery: findings from the second Gulf Registry of Acute Coronary Events. Inter Cardiovasc Thorac Surg 2012; 13(6): 611-8.
6. Elbarasi E, Goodman SG, Yan RT, et al. Management patterns of non-ST segment elevation acute coronary syndromes in relation to prior coronary revascularization. Am Heart $J$ 2010; 159(1): 40-6.

7. Wiseman A, Waters DD, Walling A, et al. Long-term prognosis after myocardial infarction in patients with previous coronary artery bypass surgery. $J$ Am Coll Cardiol 1988; 12(4): 873-80.

8. Davis KB, Alderman EL, Kosinski AS, et al. Early mortality of acute myocardial infarction in patients with and without prior coronary revascularization surgery. A Coronary Artery Surgery Study Registry Study. Circulation 1992; 85(6): 2100-9.

9. Kim MS, Wang TY, Ou F-S, et al. Association of prior coronary artery bypass graft surgery with quality of care of patients with non-ST-segment elevation myocardial infarction: A report from the National Cardiovascular Data Registry Acute Coronary Treatment and Intervention Outcomes Network Registry-Get With the Guidelines. $\mathrm{Am}$ Heart J 2010; 160(5): 951-7.

10. Teixeira R, Lourenço C, António N, et al. Can We Improve Outcomes in Patients With Previous Coronary Artery Bypass Surgery Admitted for Acute Coronary Syndrome? Rev Esp Cardiol 2010; 63(05): 554-63.

11. Gurfinkel EP, Perez de la Hoz R, Brito VM, et al. Invasive vs non-invasive treatment in acute coronary syndromes and prior bypass surgery. Int J Cardiol 2007; 119(1): 65-72.

12. Servoss SJ, Wan Y, Snapinn SM, et al. Tirofiban therapy for patients with acute coronary syndromes and prior coronary artery bypass grafting in the PRISM-PLUS trial. Am $J$ Cardiol 2004; 93(7): 843-7.

13. Peterson LR, Chandra NC, French WJ, et al. Reperfusion therapy in patients with acute myocardial infarction and prior coronary artery bypass graft surgery (National Registry of Myocardial Infarction-2). Am J Cardiol 1999; 84(11): 1287 91.

14. Labinaz M, Sketch MH, Ellis SG, et al. Outcome of acute ST-segment elevation myocardial infarction in patients with prior coronary artery bypass surgery receiving thrombolytic therapy. Am Heart J 2001; 141(3): 469-77.

15. Welsh RC, Granger CB, Westerhout CM, et al. Prior Coronary Artery Bypass Graft Patients With ST-Segment Elevation Myocardial Infarction Treated With Primary Percutaneous Coronary Intervention. JACC: Cardiovasc Interv 2010; 3(3): 343-51.

16. Alanbaei M, Alsheikh-Ali AA, Aleinati T, et al. Clinical characteristics and outcomes of patients with acute coronary syndrome and prior coronary artery bypass grafting in a large middle eastern cohort. Open Cardiovasc Med J 2011; 5: 196-202.

17. Al-Aqeedi R, Asaad N, Al-Qahtani A, et al. Acute Coronary Syndrome in Patients with Prior Coronary Artery Bypass Surgery: Observations from a 20-Year Registry in a MiddleEastern Country. PLoS ONE 2012; 7(7): e40571.

18. Bhatt DL, Roe MT, Peterson ED, et al. Utilization of early invasive management strategies for high-risk patients with non-ST-segment elevation acute coronary syndromes: results from the CRUSADE Quality Improvement Initiative. JAMA 2004; 292(17): 2096-104.

19. Goldman S, Zadina K, Moritz T, et al. Long-term patency of saphenous vein and left internal mammary artery grafts after 
coronary artery bypass surgery: Results from a Department of Veterans Affairs Cooperative Study. J Am Coll Cardiol 2004; 44(11): 2149-56.

20. Berry C, Pieper KS, White HD, et al. Patients with prior coronary artery bypass grafting have a poor outcome after myocardial infarction: an analysis of the VALsartan in acute myocardial iNfarcTion trial (VALIANT). Eur Heart J 2009; 30(12): 1450-6.

21. Suwaidi JA, Velianou JL, Berger PB, et al. Primary percutaneous coronary interventions in patients with acute myocardial infarction and prior coronary artery bypass grafting. $\mathrm{Am}$ Heart J 2001; 142(3): 452-9.

22. Al-Aqeedi RF, Al Suwaidi J, Singh R and Al Binali HA. Does prior coronary artery bypass surgery alter the gender gap in patients presenting with acute coronary syndrome? A 20-year retrospective cohort study. BMJ Open 2012; 2(6):2012-001969.

23. Brilakis ES, de Lemos JA, Cannon CP, et al. Outcomes of Patients With Acute Coronary Syndrome and Previous Coronary Artery Bypass Grafting (from the Pravastatin or Atorvastatin Evaluation and Infection Therapy [PROVE IT-TIMI 22] and the Aggrastat to Zocor [A to Z] Trials). Am $J$ Cardiol 2008; 102(5): 552-8.

24. Dittrich HC, Gilpin E, Nicod P, et al. Outcome after acute myocardial infarction in patients with prior coronary artery bypass surgery. Am J Cardiol 1993; 72(7): 507-13.

25. Kleiman NS, Anderson HV, Rogers WJ, et al. Comparison of outcome of patients with unstable angina and non-Q-wave acute myocardial infarction with and without prior coronary artery bypass grafting (thrombolysis in myocardial ischemia III registry). Am J Cardiol 1996; 77(4): 227-31.

26. Labinaz M, Kilaru R, Pieper K, et al. Outcomes of Patients With Acute Coronary Syndromes and Prior Coronary Artery Bypass Grafting: Results From the Platelet Glycoprotein
IIb/IIIa in Unstable Angina: Receptor Suppression Using Integrilin Therapy (PURSUIT) Trial. Circulation 2002; 105(3): 322-7.

27. Kugelmass AD, Sadanandan S, Lakkis N, et al. Early invasive strategy improves outcomes in patients with acute coronary syndrome with previous coronary artery bypass graft surgery: a report from TACTICS-TIMI 18. Crit Pathw Cardiol 2006; 5(3): 167-72.

28. Mitrovic PM, Stefanovic B, Vasiljevic Z, et al. In-hospital and long-term prognosis after myocardial infarction in patients with prior coronary artery bypass surgery; 19-year experience. Scient World J 2009; 9: 1023-30.

29. Mukherjee D, Gurm H, Tang WHW, et al. Outcome of acute myocardial infarction in patients with prior coronary artery bypass grafting treated with combination reduced fibrinolytic therapy and abciximab. Am J Cardiol 2002; 90(11): 1198-203.

30. Nikolsky E, McLaurin BT, Cox DA, et al. Outcomes of Patients With Prior Coronary Artery Bypass Grafting and Acute Coronary SyndromesAnalysis From the ACUITY (Acute Catheterization and Urgent Intervention Triage Strategy) Trial. JACC: Cardiovasc Interv 2012; 5(9): 919-26.

31. Neuman Y, Pereg D, Boyko V, et al. Primary angioplasty in patients following coronary artery bypass surgery. Cather Cardiovasc Intervent 2011; 78(4): 532-6.

32. Stone GW, Brodie BR, Griffin JJ, et al. Clinical and angiographic outcomes in patients with previous coronary artery bypass graft surgery treated with primary balloon angioplasty for acute myocardial infarction. J Am Coll Cardiol 2000; 35(3): 605-11.

33. Zahger D, Behar S, Harpaz D, et al. Characteristics, management and outcome of patients with prior coronary bypass surgery presenting with acute myocardial infarction. Cardiology 2003; 99(2): 105-10. 